\title{
Design of WebAccess Platform for Virtual Experiment System Based on Genetic Algorithm
}

\author{
Linlin $\mathrm{Li}^{*}$
}

Zibo Vocational Institute, Zibo, Shandong, China

\begin{abstract}
Network technology, with the popularity of computer technology and the appearance of virtual instrument technology, as a traditional experimental method and an effective way to supplement and develop virtual experiments in the field of education and other scientific fields, has very broad application prospects. Virtual experiment today has become an important direction in the development of experimental teaching. Genetic Algorithms (GA) works on the principle of natural selection and the natural genetic mechanisms based on an iterative adaptive probabilistic search method to establish the basic idea of Darwin's theory of evolution and Mendelian genetics. It is through simulation of the Darwin's "survival of the fittest" principle that the good incentive structure is excited; Mendelian genetic variation theory by simulation maintains the existing structure in an iterative process, while looking for a better structure, to achieve specific goals of artificial systems optimization. In image reconstruction, all non-redundant information is merged into an image to achieve the improvement of resolution, to achieve better recovery effect than reconstruction of a single image.
\end{abstract}

Keywords: Genetic algorithm, Virtual experiment, WebAccess platform, Remote monitoring, technology, traditional experiment.

\section{INTRODUCTION}

With the development of social economy and improvement of socialist market economy system, small and medium-sized enterprises pay more attention to the national economy and have become an important force in our society. While there exists a lot of risk in small and medium enterprises and the key risk is financial risk [1]. Especially in the depression background, the phenomenon that the overall strength is not strong with a weak point of economic risk is common in small and medium enterprises.

Experiment is one of the basic methods of scientific research catering to human understanding and different ways of understanding the world. The same experiment is an important means of teaching science and engineering; experimental teaching gives full play to an active student's initiative and creativity, As opposed to theoretical teaching, the experimental training is more conducive to students' ability, which puts great emphasis on vocational colleges link. But experimental teaching is still facing a lot of problems: experimental equipment is relatively backward and limited, laboratory equipment malfunctions and damage occur frequently which affect the conduct of regular teaching activities, thus increasing the expenditures for equipment maintenance [2]. In some of the experiments, a certain degree of risk is involved. Moreover, there are requirements of some complex and demanding conditions for the experiment which are difficult to achieve in the general lab environment. Faced with these realities, and problems that aren't easy to solve, developing virtual labs is a very effective solution.

*Address correspondence to this author at the Zibo Vocational Institute, Zibo, Shandong, China; Tel: 86- 533-2828000; E-mail: zjuyanli@163.com
In view of the present situation of the experimental teaching in colleges, the paper designs and realizes the remote teaching experimental platform based on WebAccess. This system provides a good method to solve contradiction of supply and demand of teaching resources, which has an important practical significance and application value. Advantech WebAccess is easy to use, and its friendly interface makes students to understand things visually and use conveniently [3].

In Las Vegas, at a global computer technology fair in 2007, Mr. Bill Gates, the Chairman of Microsoft, delivered a famous speech. In his speech, he proposed the incisive argument that "the only network is computer" fully reflects the important role that computer network plays in the information society. Currently, the network has infiltrated all aspects of society, such as e-commerce, e-banking, modern enterprise management, information services and so on. They all are based on the computer network system. The development of network technology provides a broad development scope for the emergence of distance education study.

\section{OVERVIEW OF DEVELOPMENT ENVIRONMENT}

Advantech WebAccess is a software that is entirely based on HMI/SCADA supervisory Control and Data acquisition Software of browser IE. WebAccess can monitor automation equipment for building automation systems or factory-made and processing control. WebAccess can read, display and store real-time data timely, and the operator can also modify the operating point, the device status, and other parameters of programmable logic controller (PLC), I/O devices and DDC (Direct Digital Control) system [4]. 


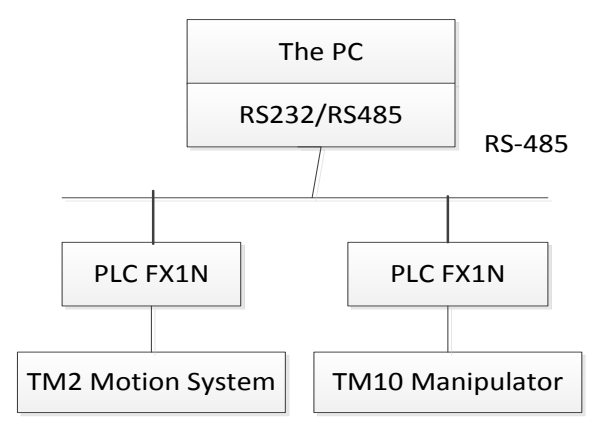

Fig. (1). Based on RS-485 and Based on CC-Link.

Advantech WebAccess constitutes a monitoring system with computer network, according to the functional allocation to each computer, to separately undertake data collection, processing, storage, management, achievement, operation, alarms, reports, trends, and other functions. These data are transmitted to each computers' subsystems, which are linked to the various automation systems via computer subsystemsa distributed control system (DCS) is formed to realize the monitoring of the whole system.

PLC control system presents a comprehensive, network development trends, in order to meet the needs of today's PLC teaching, combining the experimental teaching reform. Through Internet, a configuration software WebAccess constitutes a new remote control platform, remote control systems, remote configuration and remote access clients to monitor unlimited number of extensions [5]. The system will combine a variety of organic knowledge together to make better use of the limited teaching resources, showing the complete network control and the functions of remote monitoring technology. Practice has proved that the system is a good platform that can stimulate students' creative ability, and achieve modern, large integrated design experiments (Fig. 1).

\section{INTRODUCTION TO GENETIC ALGORITHMS}

Genetic Algorithms (GA) work on the a principle of natural selection and the natural genetic mechanisms based on an iterative adaptive probabilistic search method to establish the basic idea of Darwin's theory of evolution and Mendelian genetics. It works through simulation of Darwin's "survival of the fittest" principle to excite good incentive structure. Mendelian genetic variation theory by simulation maintains the existing structure in an iterative process, while looking for a better structure, to achieve specific goals in artificial systems optimization [6].

\subsection{The Basic Genetic Algorithm Implementation Techniques}

Different coding methods and different genetic operators constitute a variety of genetic algorithms. However, these genetic algorithms have common characteristics, i.e. selection, crossover and mutation mechanism in genetic and evolutionary process as the optimal solution for the problem of adaptive search process.

The basic genetic algorithm treats all of the individuals in a group as an object, using the three basic genetic operators: selection, crossover and mutation.Moreover, the process is simple, easy to understand and it is the foundmation of some other genetic algorithms. It not only provides a basic framework for a variety of genetic algorithms, but is also full of application value [7]. The basic genetic algorithm can be expressed as:

$S G A=\left(C, E, P_{0}, M, \Phi, \Gamma, \Psi, T\right)$

$\mathrm{C}$ expresses an individual's coding method; E expresses the individual's fitness evaluation function; $P_{0}$ expresses the initial population; $M$ expresses the population size; $\Phi$ expresses the selection of the operator; $\Gamma$ expresses the crossover; $\Psi$ expresses the mutation operator; $T$ expresses the termination condition.

\subsection{Coding Method}

A basic genetic algorithm uses the fixed-length sequence of binary symbols to represent different groups of individuals. Binary encoding is employed for mapping the solution space of the original problem into the space $\{0,1\}$, then the operator carries out genetic manipulation on bit string space, to obtain the results by decoding to restore its phenotype to evaluate fitness. The length of binary string depends on the domain of variable and the accuracy required by calculation. Assuming that the variable $\mathrm{x}$ is defined as domain $\left[\mathrm{x}_{\min }, \mathrm{x}_{\max }\right]$, variable $\mathrm{n}$ is defined as the coding length, then the accuracy of $\delta$ is:

$\delta=\frac{x_{\max }-x_{\min }}{2^{n}-1}$

Converting into a decimal number $\alpha$ according to the following formula:

$\alpha=\sum_{i=0}^{n-1} b_{j} * 2^{i}$

Calculating the corresponding variable $\mathrm{x}$ as per the following formula:

$x=x_{\min }+\frac{\alpha}{2^{n}-1}\left(x_{\max }-x_{\min }\right)$

\subsection{Fitness Function Design}

Genetic algorithms use the concept of fitness to measure each individual in the population which is likely to reach the optimization calculations or to find the optimal solution near 


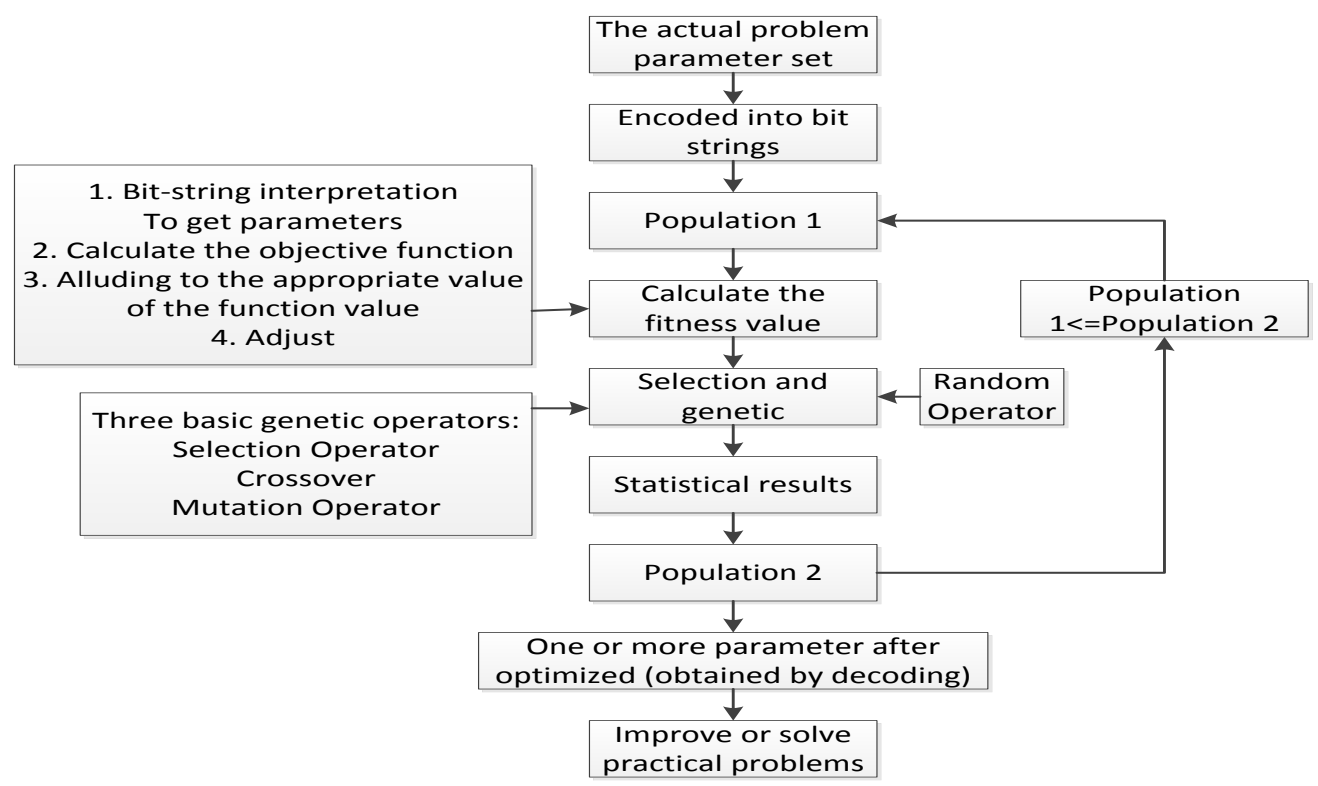

Fig. (2). Genetic Algorithm for Computing Processes.

the fine degree. From mathematical point of view, the genetic algorithm is essentially a probabilistic search algorithm, but it also has some smart features. The basic genetic algorithm according to the probability of individual fitness is proportional to the current population of each individual genetic group with opportunities for the next generation number. In order to correctly estimate the probability, all of the individuals' fitness must be nonnegative. So, depending on the type of problem, we need to determine the laws of converting the objective function into individual fitness.

\subsection{Genetic Manipulation}

Genetic manipulation is an operation that simulates the biological genetics. The basic genetic algorithm uses the following three basic genetic operators: selection, cross, and variation.

The operation of selection simulates the Laws of natural selection in the biological evolution. It is a process that selects the vitality of the individual from an older population to generate a new population. The basic idea is that each individual's probability of being selected is proportional to its fitness. Assuming that the population size is $M$, and the fitness of individual $i$ is $f_{i}$, then the probability of individual $i$ being selected as follows:

$P_{i}=f_{i} / \sum_{k=1}^{M} f_{k}$

\subsection{Genetic Algorithm Flow}

Full genetic algorithm flow can be seen in Fig. (2):

Genetic algorithms can be seen from the operation process. The evolutionary process is simple and easy to understand, and it provides a basic framework for a variety of other genetic algorithms.

\section{FUNCTIONAL DESIGN}

The user can set the appropriate experimental parameters in an experimental interface. After making a good selection of laboratory equipment, the parameters will be returned to the remote Web server. The Web server sends the parameters to the embedded controller LAN simultaneously. The embedded controller is used for designating the results of sampling and sends back to the Web server by the control of the machine. Following this, the Web server sends the results to the client in real-time in the form of dynamic pages. Customers can see the results in real-time on-line and adjust the experimental parameters to achieve the purpose of the experiment [8-12].

After obtaining the variable, the browser carries out analysis and uses the technology of DOM to locate the elements that need to be changed in the SVG image, making the corresponding changes to the corresponding image elements according to changes in the analysis of information obtained. Following this, the display of a dynamic image in real-time is achieved (Fig. 3).

The paper not only develops a virtual lab which possesses advantages such as relatively free schedule and place compared with the practical experiment, but also provides a virtual research platform for the application of the advanced control theory. In the initial equilibrium conditions, by changing the opening of the electric control valve, the step disturbance is applied to the system. We can see from that after the system has reached the initial steady state operating point in the initial conditions, the rise and fall for the relatively large space, indicate that the system receives a step disturbance, and its steady-state value can be varied within a range close to full scale(Fig. 4). 


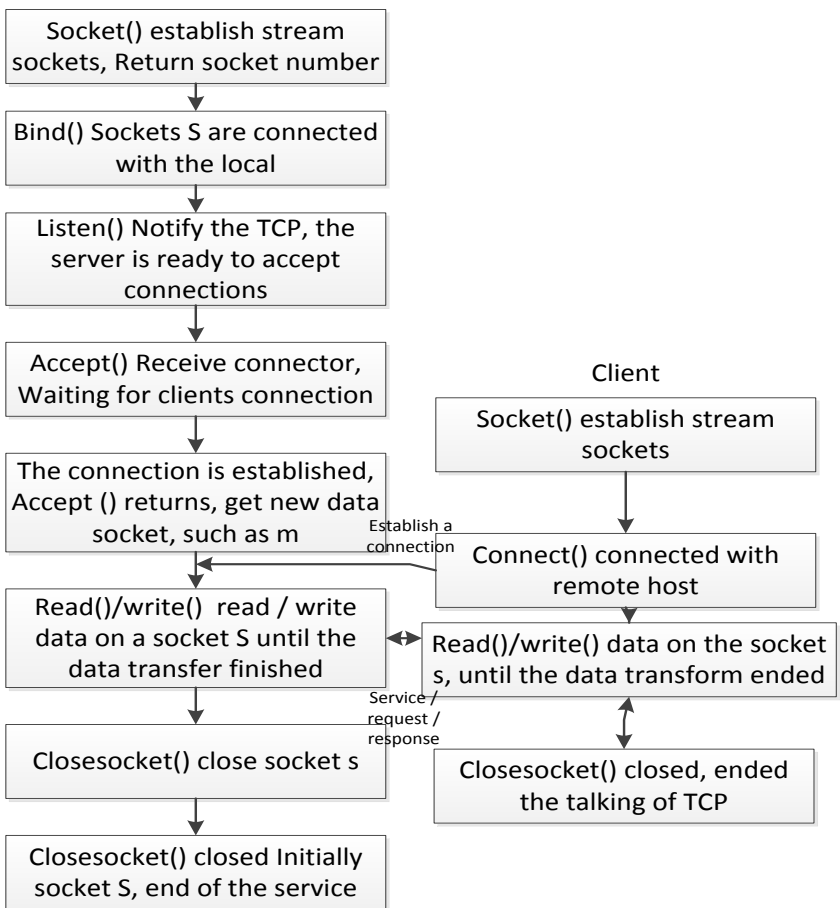

Fig. (3). Using the TCP Protocol Client / Server Mechanism.

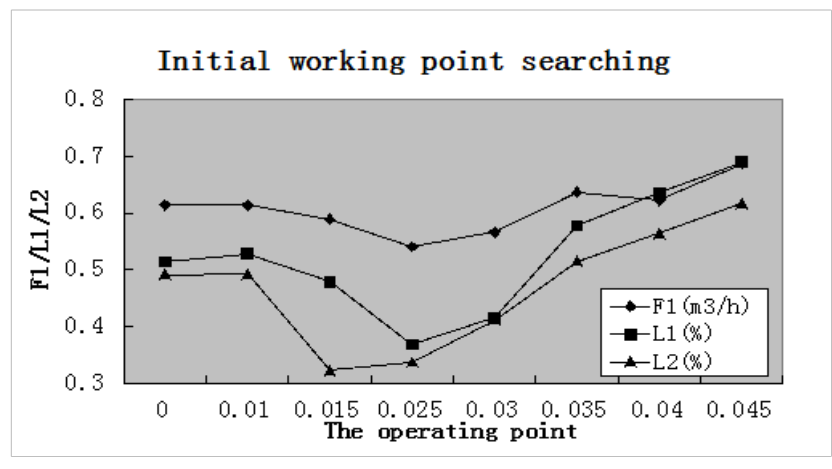

Fig. (4). Initial working point searching.

\section{CONCLUSION}

With the continuous development of computer technology and advances of network technology, information technology has penetrated in all aspects of society. People's lifestyles are undergoing unprecedented changes. In the field of education, information and networking have become a useful supplement to traditional education, information sharing and its features make quick calculations that have significantly changed the traditional mode of education, improved the efficiency of society and the learning efficiency. Experiment as an important part of teaching activities, is an important way for students to acquire knowledge, but also an important means to improve the quality of students. But there are many actual problems we cannot solve effectively, so how to achieve resource sharing of laboratory equipment becomes a problem to carry out network experiments that needs to be solved urgently. Practice has proved that Advantech WebAccess can quickly complete the development and design work, and the monitoring system is easy to install, maintain and use, having good compatibility, high reliability and good effects.

\section{CONFLICT OF INTEREST}

The authors confirm that this article content has no conflict of interest.

\section{ACKNOWLEDGEMENTS}

Declared none.

\section{REFERENCES}

[1] W. Anderson, "Learning to Control an Inverted Pendulum Using Neural Network," IEEE Control System Magazine, vol. 9, no.4, pp.3137, 1989 .

[2] B. H. Freyer, LK. Craig, and P. C. Pistorius, "Gauge and Tension Control during the Acceleration Phase of a Sticker Hot Rolling Mill," AFRICON, IEEE, vol. I, pp. 425-430, 1999.

[3] S.C. Brofferio, "A university distance lesson system: experiments, services, and future developments," IEEE Trans. Educ, vol.41, pp.17-24, 1998.

[4] E. Scholtz, L. Z. Craig, and P. C. Pistorius, "Modeling for control of a Steckel hot rolling mill," ISIJ International, vol. 40, no.10, pp.1003-1012, 2000.

[5] L. Guo, N. Li, and Y. Guo, "The Virtual Lab System," The Journal of China Universities of Posts And Telecommunications, vol. 10, no.2, pp.81-84, 2003.

[6] C. C. Ko, B. M. Chen, J. Chen, and K. C. Tar, "Development of a Web-based laboratory for control experiments on a coupled tank apparatus," IEEE Transactions on Education, vol. 44, no.1, pp.76$86,2001$.

[7] S. Mori, H. Nishihara, and K. Furuta, "Control of Unstable Mechanical System Control of Pendulum," Control, vol. 23, pp. 673692, 1975.

[8] H. Y. Li, "Web-Based Remote Monitoring and Control for Process Plants," Machine Learning and Cy2bernetics, Proceedings of 2005 International Conference, vol. 2, no.18-21, pp.936-941, 2005.

[9] R. Singh, "Developing Models from Experimental Data using System Identification Toolbox," [EB/OL] 2007.

[10] L. Xu, L. Cheng, "Industry Control Machine and Network Control System," China Machine Press, 2005.

[11] Z. Xin, J. Zhang, and X. Luo, "Tuning PID Parameters Based on a Combination of the Expert System and the Improved Genetic Algorithms," Petroleum Science, vol.2, no.4, pp. 71-76, 2005.

[12] T. Yamakwa, "Stabilization of an Inverted Pendulum by a Highspeed Fuzzy Logic Controller Hardware System," Fuzzy Sets and Systems, vol. 32, pp.161-180, 1975.

\footnotetext{
Received: June 10, 2015

Revised: July 29, 2015

Accepted: August 15, 2015

(C) Linlin Li; Licensee Bentham Open.

This is an open access article licensed under the terms of the (https://creativecommons.org/licenses/by/4.0/legalcode), which permits unrestricted, noncommercial use, distribution and reproduction in any medium, provided the work is properly cited.
} 